\title{
Social network analysis of scientific collaborations across different subject fields
}

\author{
Hamparsum Bozdogan* and Oguz Akbilgic \\ Department of Statistics, Operations, and Management Science, The University of Tennessee, \\ Knoxville, TN 37996, USA \\ E-mails:bozdogan@utk.edu,oguzakbilgic@gmail.com
}

\begin{abstract}
This paper analyzes the level of scientific collaboration and interaction in different subject fields using a novel social network analysis (SNA) on a data set provided by the Department of Energy (DOE) Office of Scientific and Technologic Information (OSTI) in Oak Ridge, Tennessee. This paper not only determines the level of scientific collaboration between different disciplines, but it also analyzes the trends among the subject fields considered. The results in this paper show clear pattern recognition discovery on the social network model among key subject fields using weighted networks.
\end{abstract}

Keywords: Social network analysis, weighted networks, scientific collaboration

\section{Introduction}

This study is carried out as a joint project between the University of Tennessee (UT) and Information International Associates, Inc. (IIa) under the grant entitled "Exploiting the Use of Social Networking to Facilitate Collaboration in the Scientific Community", which was awarded by the Department of Energy (DOE) Experimental Program to Stimulate Competitive Research (EPSCoR). The authors, Dr. Bozdogan and Dr. Akbilgic, serve as the grant's Principle Investigator and Post-Doctoral Research Associate, respectively.

Social networks are systems consisting of two elements: nodes and edges between nodes. For the purposes of this analysis, nodes can be individuals, companies, countries, etc., while edges refer to the interaction or relation between the nodes. The objective of social network analysis (SNA) is to examine the structure of the network and to analyze the relations between the nodes within the network using graph theory based statistical techniques. The paper does not attempt to provide any analysis of the changes that are driven by the subject matter or by technology.

The SNA techniques described herein are applied in many different research areas, especially in sociology and social and behavioral sciences. In related research literature, Tichy et al. [24] applies SNA to organizations, while Johnson et al. [12] uses social networks to study friendship and communication structure between groups of people working in the same bank by analyzing email trafficking data. Pow et al. [20] suggests usage of SNA in nursing to analyze why some people are open to organizational changes while the others are not. Similarly, SNA is used and applied further by many other authors such as Quinn et al. [21], Mainas [13], Oshima et al. [19] and Cserpes [7].

\footnotetext{
*Corresponding author. Tel.: +1 865974 1635; Fax: +1 865974 2490; E-mail: bozdogan@utk.edu.
} 
In scientific collaborations, Hossain and Fazio [11] conducted a study using SNA to analyze interactions between academics who developed the publications in Web of Science. In their model, the nodes are represented by the authors who published the papers, and the edges are represented by the papers. In medical sciences, Eblen et al. [9] use SNA to analyze collaborations among researchers in two cardiovascular cohort studies.

In this study, we analyze the data collected and organized by DOE OSTI to determine the level of scientific collaboration between different subject areas or fields of study. OSTI is based in Oak Ridge, Tennessee, and supports DOE in the collection, preservation, and dissemination of DOE-sponsored research and development (R\&D) results. For more information on OSTI data, we refer the readers to the OSTI Information Bridge website at http://www.osti.gov/bridge/ [8]. For this study, we used all the data in the information Bridge database, which contains DOE R\&D full-text documents and bibliographic citations. Analyzing the OSTI database involves (1) determining the level of scientific collaboration between different disciplines, and (2) discovering the trends of different research interests supported by DOE.

Using the OSTI database we used SNA to determine levels of scientific collaboration amongst different disciplines and related trends in collaboration to the research interests supported by DOE. Our research was constrained by factors such as publication ID, publication date, and number of authors per publication, author names. However, in reviewing the SNA literature, we note that this paper is unique in its contribution using subject fields as network nodes connected by co-authorship across the subject fields.

This paper is organized as follows:

- In Section 2, we present the summary results of SNA and the necessary definitions and network metrics.

- In Section 3, we present and introduce the OSTI database, the results from our analysis, including the descriptive statistics, the SNA, and the weighted SNA to construct the structure of the network.

- Finally, in Section 4, we summarize the analysis and conclude the paper.

\section{Social Network Analysis (SNA)}

For purposes of this research, we define the social network as follows: (Referencing Wasserman and Faust [25]),

"A social network is a social structure [27] made up of a set of actors (such as individuals or organizations) and the dyadic ties [28] between these actors. The social network perspective provides a clear way of analyzing the structure of whole social entities".

In a social network, relations can be any of the gained or defined ties that can be discovered among individuals. Often, individuals are also called nodes, while the relations between nodes are called edges (Bandyopadhyay et al. [1]). In our research, we will refer to nodes as subject fields, and edges as the relations (co-authorship with publication) between nodes.

Social network analysis (SNA) refers to the statistical techniques to analyze social networks based on graph and network theory, which is explained in detail in Wasserman and Faust [25]. SNA techniques include analyzing the nodes, the edges, and the entire network according to special measures. Connectivity and centrality measures are the most commonly used network statistics. Connectivity measures indicate the extent to which the nodes are connected to each other in the network. Here, higher con- 
nectivity indicates higher information flow between the nodes in the network. Since in our study, the subject fields are considered as nodes, connectivity measures will show how strongly different subject fields are connected. Furthermore, the interpretation of connectivity depends on the type of the nodes and relations between the nodes in the network. Detailed discussion of these can be found in Bavelas [3] and Wassermann and Steinley [26].

Centrality measures indicate the importance of individual nodes. Centrality measures help the researcher to determine which nodes are important to be kept in the network. The literature references several types of centrality measures. For example, according to Freeman [10], there are three major centrality measures: degree, closeness and betweenness. In our study, centrality measures will reflect the importance of specific subject fields in terms of scientific collaboration via co-authorship.

Degree centrality measures the direct connection of an individual node in the network (Schneider et al. [22]). As a node's number of edges increases, its degree centrality also increases. Betweenness centrality of a node measures togetherness of nodes in the network. Finally, closeness centrality measures how fast the information spreads from a given node to other reachable nodes in the network (Maslow and Sneppen [14]). According to given definitions of degree centrality measures, in our study, collaboration between two specific subject fields will be measured by centrality measures.

Determining centrality measures for nodes in a network is one of the most important problems in SNA (Freeman [10], Bonacich [4] and Borgatti [5]). Freeman's [10] three centrality measures are to be applied to binary networks. However, several studies have been conducted on the generalization of centrality measures to weighted networks (Brandes [6], Newman [15,16], Barrat et al. [2]). While these authors focus on the weights of the edges, Opsahl et al. [18] focus on the number of edges between nodes. In this study, we use betweenness and closeness centrality measures, as suggested by Opsahl et al. [18]. Calculations of related measure are based on the information given by Table 2. Network diagrams in Figs 5 and 6 are also derived from Table 2.

\section{Social Network Analysis (SNA) of OSTI database}

DOE OSTI collects and organizes the records of scientific studies and collaborations, which are fully or partially supported by DOE. The OSTI database includes metadata records for scientific technical reports such as author names, publication title, identification number, publication date, first and last publication dates of the authors, publication span, author affiliation, subject category, etc. In our study, we considered attributes, which include author names, publication identification, publication date, author's publication span, and subject categories. The database in this study contained 256,320 publications, 265,381 authors, and 827,614 author - publication links collected for the time period from 1920 to 2012.

First we produced the descriptive statistics (such as number of authors, number of publications, average number of authors per publication, and average number of papers per author for one year) of overall OSTI data to get an idea of author productivity and level of collaboration for each subject field. Second, we determined the trends in descriptive statistics for different time periods for each subject field. Then, SNA was carried out to analyze the scientific collaborations across different subject fields.

\subsection{Descriptive statistics for OSTI data}

In this section, we provide general information about the data under consideration for different scientific subject areas contained in the OSTI data. Because there are hundreds of different subject fields 
Table 1

Summary of different subject fields of science (1920-2012)

\begin{tabular}{|c|c|c|c|c|}
\hline Subject fields & $\mathrm{NP}^{1}$ & $\mathrm{NA}^{2}$ & ANAPP $^{3}$ & ANPPA1Y ${ }^{4}$ \\
\hline Astronomy and space & 2,440 & 4,706 & 3.05 & 0.69 \\
\hline Chemistry & 16,838 & 23,958 & 2.85 & 0.53 \\
\hline Energy & 33,409 & 53,413 & 3.30 & 0.77 \\
\hline Engineering & 17,775 & 32,450 & 2.96 & 0.55 \\
\hline Environment & 22,821 & 35,965 & 3.32 & 0.66 \\
\hline Fossil fuel & 27,698 & 33,983 & 3.30 & 0.94 \\
\hline Geosciences & 2,711 & 5,571 & 3.18 & 0.58 \\
\hline Life sciences & 10,456 & 16,771 & 3.15 & 0.52 \\
\hline Material science & 9,503 & 17,761 & 3.31 & 0.49 \\
\hline Math and computation & 6,222 & 12,702 & 3.23 & 0.72 \\
\hline Military and weapon & 3,585 & 7,435 & 3.34 & 0.65 \\
\hline Miscellaneous & 2,216 & 4,609 & 2.86 & 0.55 \\
\hline Nuclear & 47,327 & 63,797 & 3.36 & 0.65 \\
\hline Particle acceleration & 4,240 & 9,489 & 3.91 & 0.36 \\
\hline Physics & 36,021 & 48,795 & 3.29 & 0.39 \\
\hline
\end{tabular}

${ }^{1}$ Number of Publications (NP): Number of reports published in each subject field.

${ }^{2}$ Number of Authors (NA): Number of authors with at least one publication in the subject field under consideration. Those with publication(s) in more than one subject field are counted separately for each subject field.

${ }^{3}$ Average Number of Authors per Publication (ANAPP): Average number of authors for publications, calculated separately for each subject field. This statistic is directly related to the level of collaboration between scientists.

${ }^{4}$ Average Number of Papers per Author for One Year (ANPPA1Y): For a specific subject field, ANPPA1Y is the average of the ratios obtained by dividing the number of an author's publications over the span of time. This statistic gives the expected number of publications for a scientist for the subject field under consideration. The reason the ANPPA1Y statistic is included in this study is to compare the productivity of scientists from different subject fields.

in the OSTI database, we grouped the subject fields into broader categories with regard to similarity of the research areas, reducing the number of subject fields to 15 . We grouped all the other papers as miscellaneous. The first column in Table 1 represents the list of subject fields, while the other columns provide information on descriptive statistics for the data between 1920 and 2012.

Table 1 shows that the Average Number of Authors per Publication (ANAPP) statistic varies from 2.85 to 3.91. This means that, a publication in chemistry is written by 2.85 authors on the average, while the average number of authors is 3.91 for a publication on particle acceleration. Further, the ANAPP statistic shows the strength of collaboration of the scientists in the particle acceleration, nuclear, military and weapon, material sciences, energy, and fossil fuels fields as compared to other subject fields.

Figure 1 shows the variation of the rounded ANAPP statistic for different subject fields in ranked order for the data from 1920 to 2012.

Average Number of Papers per Author for One Year (ANPPA1Y) statistic is calculated as a ratio of the total number of publications and the publication span of authors. An author, who has only one written paper, where the publication span is zero, is excluded from the calculations for this statistic. ANPPA1Y is the measure of the expected number of papers per author for one year. Therefore, ANPPA1Y is directly related to author productivity. The ANNPA1Y statistic varies from 0.36 for the field of particle acceleration to 0.94 for the field of fossil fuels. The ANNPA1Y statistic for all subject fields indicates the productivity of the authors from fossil fuels, energy, and math and computation, which is higher 


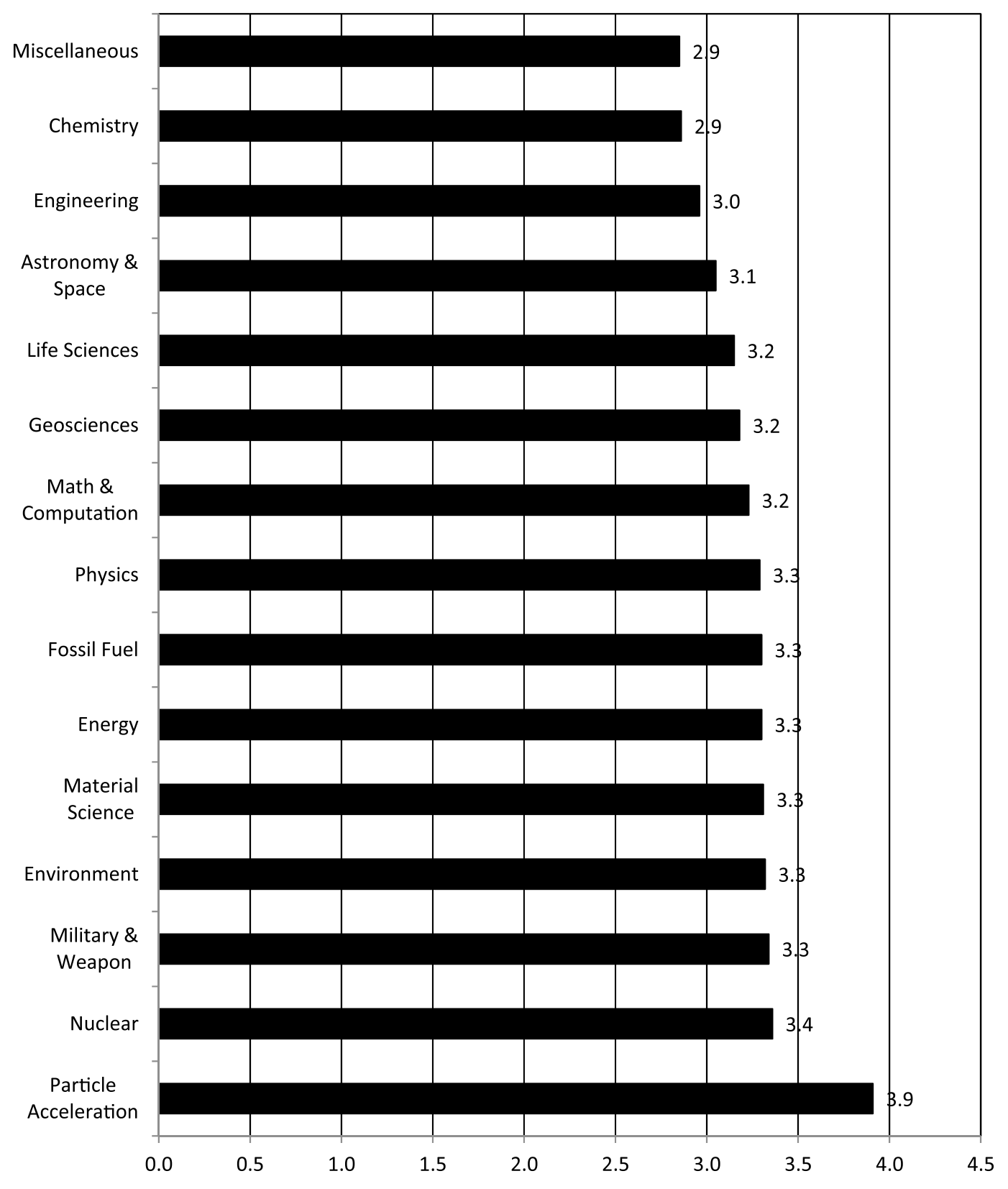

Fig. 1. Variation of ANAPP statistics for different subject fields.

than the productivity of the authors from the subject fields of particle acceleration and physics. Figure 2 shows the ANPPA1Y statistic for different subject fields in descending order.

Figure 2 shows that an author in fossil fuels publishes almost one paper per year, while an author in particle acceleration publishes 0.36 papers per year (i.e., one paper for every 2.5 years). Hence, Fig. 2 can be a yardstick to compare the productivity of the authors in different subject fields. 


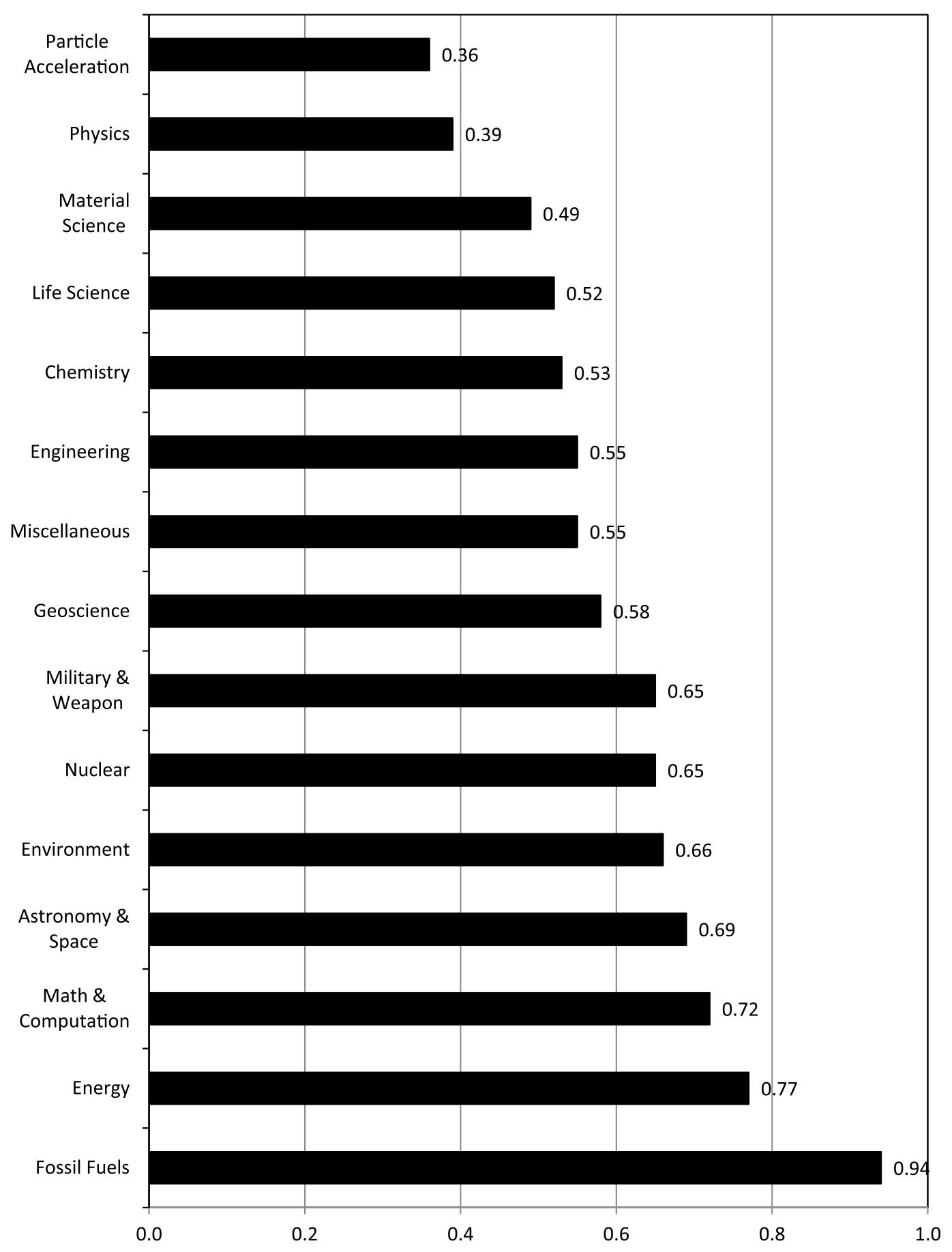

Fig. 2. Scientists productivity from different subject fields. 


\subsection{Analyzing the trends in ANAPP and ANPPA1Y statistics}

Section 3.1 above presents descriptive statistics calculated from overall data for each subject field. In this section, we monitor the trends of the ANAPP and ANPPA1Y statistics for different time periods. For this purpose, the data are split into five different time periods: before 1940, 1941-1960, 1961-1980, 1980-2000 and 2000-2012 in order to have balanced number of publications in each time period. ANAPP and ANPPA1Y statistics are calculated for each time period, as shown in Figs 3 and 4, respectively.

Figure 3 shows that the ANAPP statistic tends to increase over time for almost all subject fields, increasing most dramatically in the last 12 years. This positive trend in the ANAPP statistic can be considered as evidence of increasing collaboration between scientists. This trend can be explained by fast development of new technologies and increasing usage of internet, which makes distance collaboration easy between scientist.

Although Fig. 4 does not reflect any common trend for the ANPPA1Y statistic across all disciplines, scientists' productivity in the last 12 years seems to be higher in comparison to earlier time periods. In the past 12 years, dramatic increases in scientists' productivity can be seen particularly in fossil fuels, material science, engineering, and military and weapon.

\subsection{Social Network Analysis (SNA) of OSTI data}

In this study, we used Cytoscape software (Version 2.8.3) to carry out the SNA of the OSTI data set. Cytoscape is open source software originally used for the analysis and visualization of biological networks in genome research, originally introduced by Shannon et al. [23]. Although the focus of Cytoscape is for biological networks, most of the computations of network statistics are applicable for other social network modeling problems. In addition to Cytoscape, we also used the tnet package of R software from Opsahl [17] to calculate and construct the weighted networks.

In social network modeling of OSTI data, subject fields are considered as single nodes, while authors are connectors (or links) between subject fields. For instance, an author having two publications, one in material science and one in physics, creates a link between the fields of material science and physics. OSTI data are preprocessed to expose authors having publications in more than one area so that the links between subject fields can be monitored. To be able to see the structural changes of scientific collaboration across time periods, we constructed two separate social network models of subject fields for two different time periods during 1980-2000 and 2000-2012. In each cell of Table 2, the upper and lower numbers show the number of authors having publications in the intersection of two subject fields for the time periods during 1980-2000 and 2000-2012, respectively.

There are different ways to interpret the cell frequencies in Table 2. For example, the frequencies at the intersection of the energy subject field are much greater than the frequencies in other subject fields. This means that, in the energy subject field, a research study requires more knowledge background from other disciplines. Energy studies bring scientists together to work on the same projects from different subject fields. Moreover, there are not many bridge authors between certain disciplines, such as the intersection of geosciences - particle acceleration and astronomy and space-military and weapon, etc.

The raw frequencies shown in Table 2 can be considered as the strength of collaboration between two subject fields. Since the duration of the two time periods in the Table 2 are not equal, it is not convenient to compare the level of collaboration between subject fields directly using the raw frequencies in Table 2 . However, we can visualize the networks separately for the two time periods by constructing a weighted 


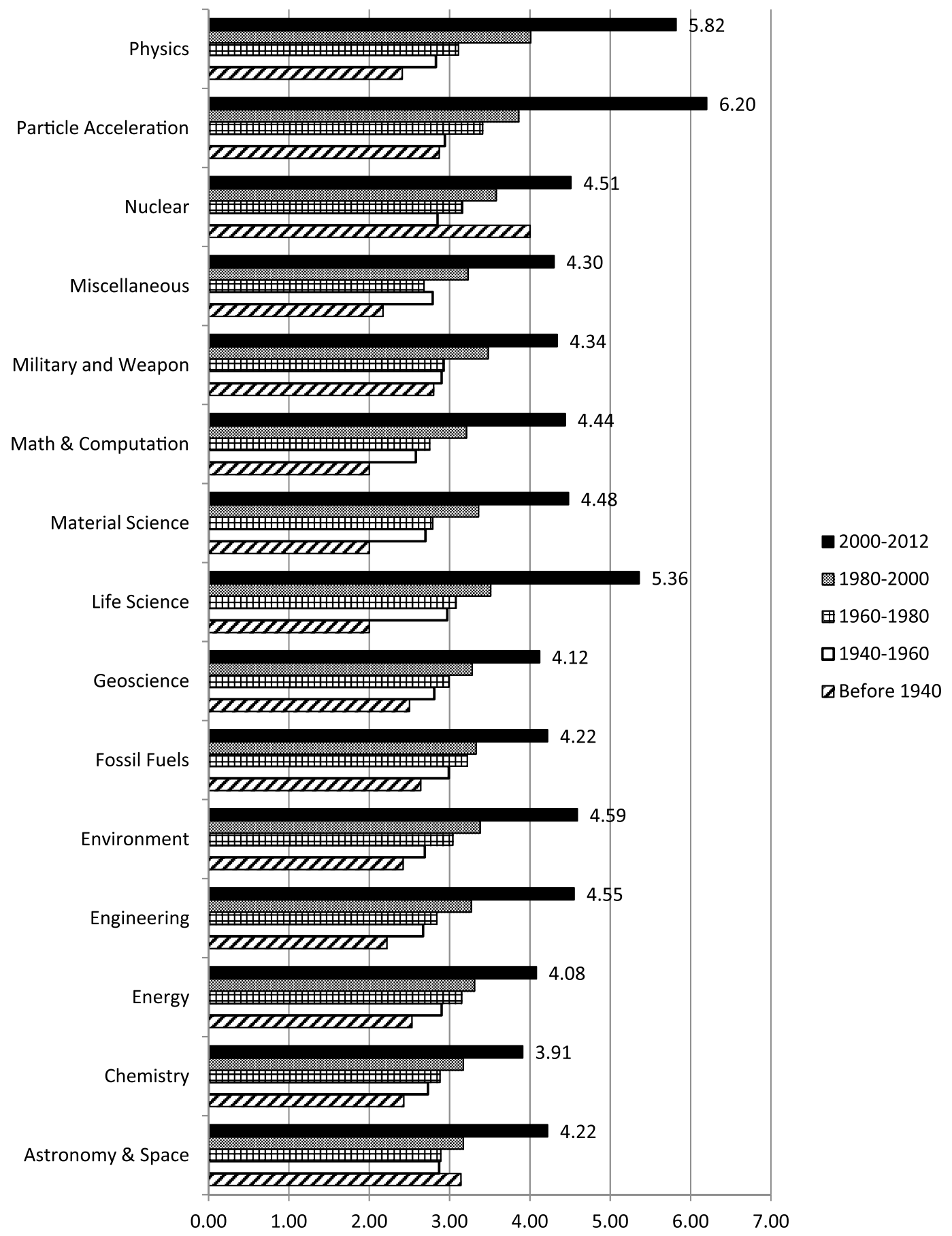

Fig. 3. Average number of authors per publication. 


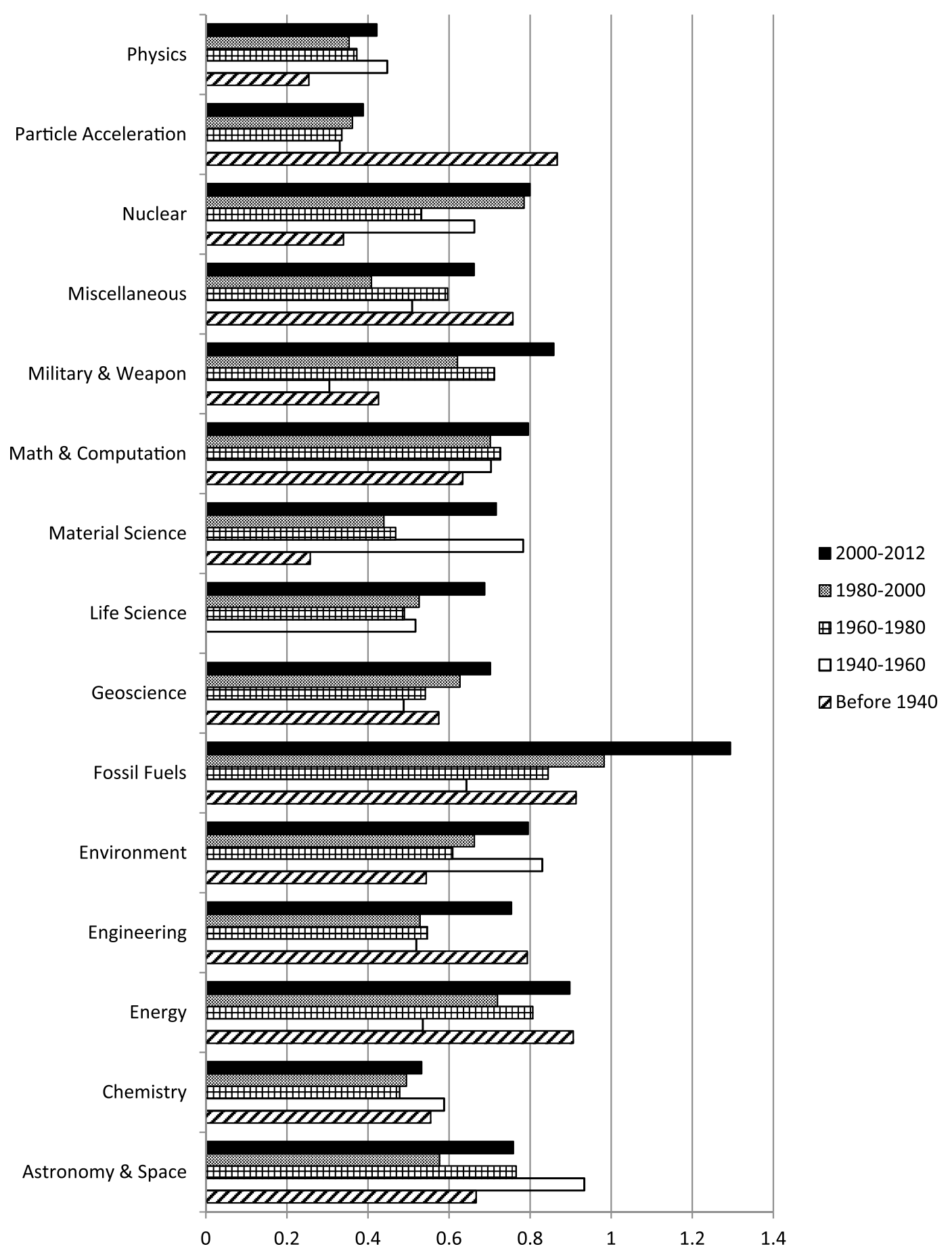

Fig. 4. Average number of papers per author for 1 year. 
Table 2

Frequency of DOE information bridge authors between subject fields (1980-2000 upper line, 2000-2012 lower line)

\begin{tabular}{|c|c|c|c|c|c|c|c|c|c|c|c|c|c|c|c|}
\hline & $\begin{array}{l}\text { Astronomy } \\
\text { and space }\end{array}$ & Chemistry & Energy & $\begin{array}{c}\text { Engi- } \\
\text { neering }\end{array}$ & $\begin{array}{c}\text { Envi- } \\
\text { ronment }\end{array}$ & $\begin{array}{l}\text { Fossil } \\
\text { fuels }\end{array}$ & $\begin{array}{l}\text { Geo- } \\
\text { science }\end{array}$ & $\begin{array}{c}\text { Life } \\
\text { sciences }\end{array}$ & $\begin{array}{c}\text { Material } \\
\text { science }\end{array}$ & $\begin{array}{c}\text { Math and } \\
\text { compu- } \\
\text { tation }\end{array}$ & $\begin{array}{c}\text { Military } \\
\text { and } \\
\text { weapon }\end{array}$ & $\begin{array}{l}\text { Miscel- } \\
\text { laneous }\end{array}$ & Nuclear & $\begin{array}{c}\text { Particle } \\
\text { acceler- } \\
\text { ation }\end{array}$ & Physics \\
\hline \multirow{2}{*}{$\begin{array}{l}\text { Astronomy } \\
\text { and space }\end{array}$} & \multirow[t]{2}{*}{-} & 45 & 276 & 41 & 94 & 89 & 0 & 1 & 44 & 5 & 6 & 0 & 20 & 3 & 10 \\
\hline & & 4 & 72 & 10 & 8 & 68 & 0 & 4 & 10 & 4 & 2 & 2 & 4 & 4 & 12 \\
\hline \multirow[t]{2}{*}{ Chemistry } & \multirow[t]{2}{*}{-} & \multirow[t]{2}{*}{-} & 421 & 210 & 344 & 199 & 17 & 52 & 189 & 31 & 45 & 1 & 283 & 9 & 49 \\
\hline & & & 122 & 58 & 110 & 28 & 12 & 44 & 82 & 42 & 12 & 2 & 86 & 0 & 20 \\
\hline \multirow[t]{2}{*}{ Energy } & \multirow[t]{2}{*}{-} & \multirow[t]{2}{*}{-} & \multirow[t]{2}{*}{-} & 821 & 2080 & 1255 & 61 & 114 & 528 & 253 & 106 & 6 & 614 & 52 & 178 \\
\hline & & & & 282 & 594 & 642 & 24 & 116 & 400 & 228 & 62 & 34 & 394 & 106 & 120 \\
\hline \multirow[t]{2}{*}{ Engineering } & \multirow[t]{2}{*}{-} & \multirow[t]{2}{*}{-} & \multirow[t]{2}{*}{ - } & \multirow[t]{2}{*}{-} & 566 & 428 & 48 & 60 & 615 & 242 & 168 & 5 & 501 & 97 & 240 \\
\hline & & & & & 96 & 82 & 36 & 74 & 314 & 158 & 42 & 32 & 120 & 62 & 74 \\
\hline \multirow[t]{2}{*}{ Environment } & \multirow[t]{2}{*}{-} & \multirow[t]{2}{*}{-} & \multirow[t]{2}{*}{ - } & \multirow[t]{2}{*}{ - } & \multirow[t]{2}{*}{-} & 1560 & 300 & 478 & 292 & 297 & 277 & 4 & 1591 & 47 & 171 \\
\hline & & & & & & 294 & 206 & 104 & 142 & 134 & 100 & 60 & 494 & 14 & 34 \\
\hline \multirow{2}{*}{$\begin{array}{l}\text { Fossil } \\
\text { fuels }\end{array}$} & \multirow[t]{2}{*}{-} & \multirow[t]{2}{*}{-} & \multirow[t]{2}{*}{-} & \multirow[t]{2}{*}{ - } & \multirow[t]{2}{*}{-} & \multirow[t]{2}{*}{-} & 182 & 141 & 514 & 210 & 144 & 4 & 631 & 51 & 136 \\
\hline & & & & & & & 100 & 26 & 156 & 58 & 18 & 6 & 128 & 14 & 56 \\
\hline Geoscience & - & - & - & - & - & - & - & 26 & 98 & 63 & 103 & 0 & 338 & 3 & 24 \\
\hline & & & & & & & & 14 & 52 & 58 & 30 & 0 & 166 & 2 & 12 \\
\hline Life & - & - & - & - & - & - & - & - & 85 & 94 & 76 & 3 & 274 & 7 & 45 \\
\hline science & & & & & & & & & 172 & 108 & 34 & 10 & 60 & 10 & 14 \\
\hline Material & - & - & - & - & - & - & - & - & - & 221 & 172 & 10 & 986 & 90 & 481 \\
\hline science & & & & & & & & & & 142 & 118 & 60 & 576 & 62 & 178 \\
\hline Math and & - & - & - & - & - & - & - & - & - & - & 252 & 9 & 731 & 98 & 251 \\
\hline computation & & & & & & & & & & & 172 & 70 & 256 & 96 & 100 \\
\hline Military and & - & - & - & - & - & - & - & - & - & - & - & 6 & 589 & 30 & 140 \\
\hline weapon & & & & & & & & & & & & 26 & 202 & 12 & 64 \\
\hline Miscellaneous & - & - & - & - & - & - & - & - & - & - & - & - & 37 & 5 & 19 \\
\hline & & & & & & & & & & & & & 96 & 6 & 40 \\
\hline Nuclear & - & - & - & - & - & - & - & - & - & - & - & - & - & 412 & 1009 \\
\hline & & & & & & & & & & & & & & 246 & 434 \\
\hline $\begin{array}{l}\text { Particle } \\
\quad \text { acceleration }\end{array}$ & - & - & - & - & - & - & - & - & - & - & - & - & - & - & $\begin{array}{l}539 \\
632\end{array}$ \\
\hline Physics & - & - & - & - & - & - & - & - & - & - & - & - & - & - & - \\
\hline
\end{tabular}


social network between different subject fields. Separate network visualization allows us to compare the structure of collaboration between different subject fields.

The information in Table 2 is converted to a related data form in order to visualize the social networks in Cytoscape. The frequencies in Table 2 are considered as edge weights in the social network model. Weights are associated with the darkness of the edge color, changing from white to black. Darker edges indicate higher collaboration compared to lighter colored edges. In this respect, Figs 5 and 6 show the structure of collaborations among subject fields for time periods 1980-2000 and 2000-2012, respectively.

Figure 5 shows which subject fields are in close collaboration for the time period 1980-2000. By following the darker edges, we can definitely see a pattern structure naturally emerging from the data. One can easily see how strongly the environment subject field is related to the nuclear (average 80 connection per year), energy (average 106 connection per year) and fossil fuels (average 78 connection per year) subject fields. By taking the somewhat less dark edges into account, we can see the high level of collaboration between the environment, nuclear, energy, fossil fuels and physics subject fields.

From the results of our analysis, Fig. 6 shows the social network diagram of subject fields for the time period of 2000-2012. Comparing Figs 5 and 6, we see that there are some major changes in the structure of social network among subject fields from 1980-2000 to 2000-2012. One of the most prominent changes is the increasing level of collaboration between the physics and particle acceleration (average number of connections goes up from 27 to 53) fields. Another important change in the network diagram is that there is a notable jump on the collaboration between the nuclear subject field and the others, especially material science and energy. On the contrary, the collaboration level between environment and fossil fuels subject fields has declined in the last 12 years compared to the time period of 1980-2000. Additionally, the level of collaboration between the engineering subject field and other subject fields such as energy, environment, and material science are slightly decreased in the last 12 years.

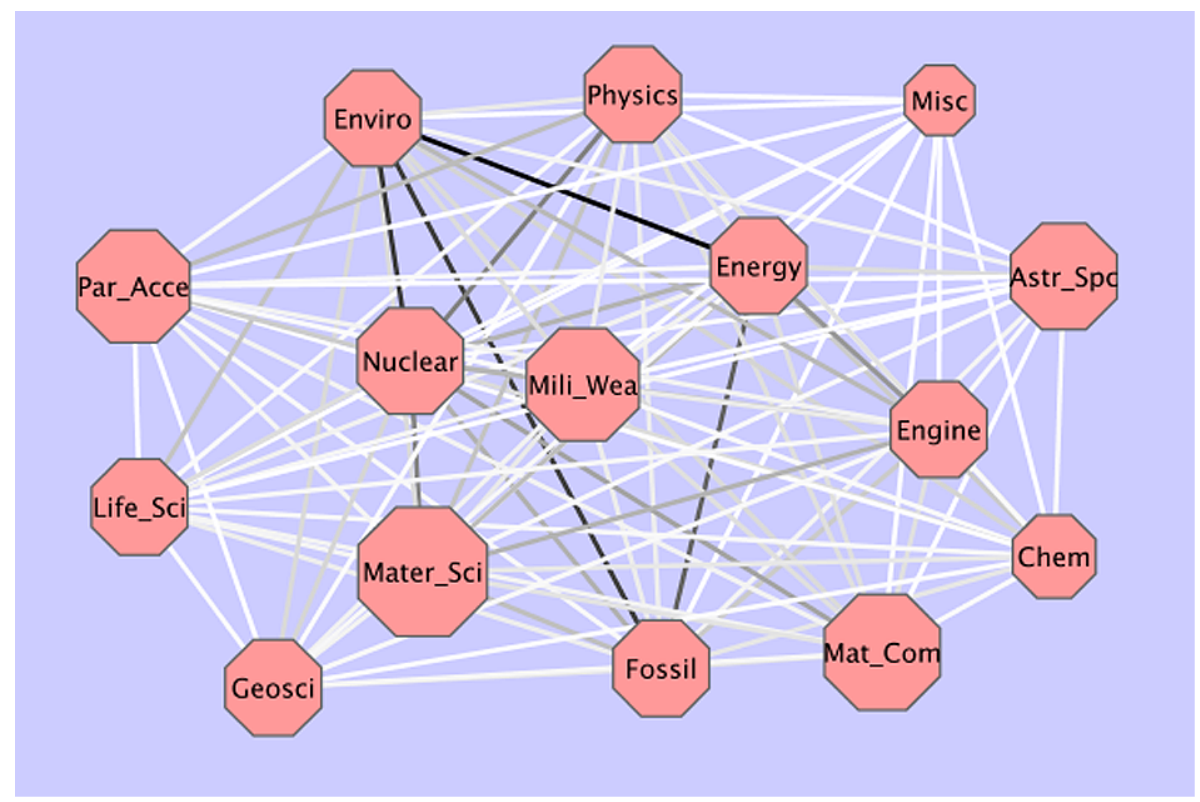

Fig. 5. Social network of subject fields during the time period 1980-2000. (Colors are visible in the online version of the article; http://dx.doi.org/10.3233/ISU-130715.) 


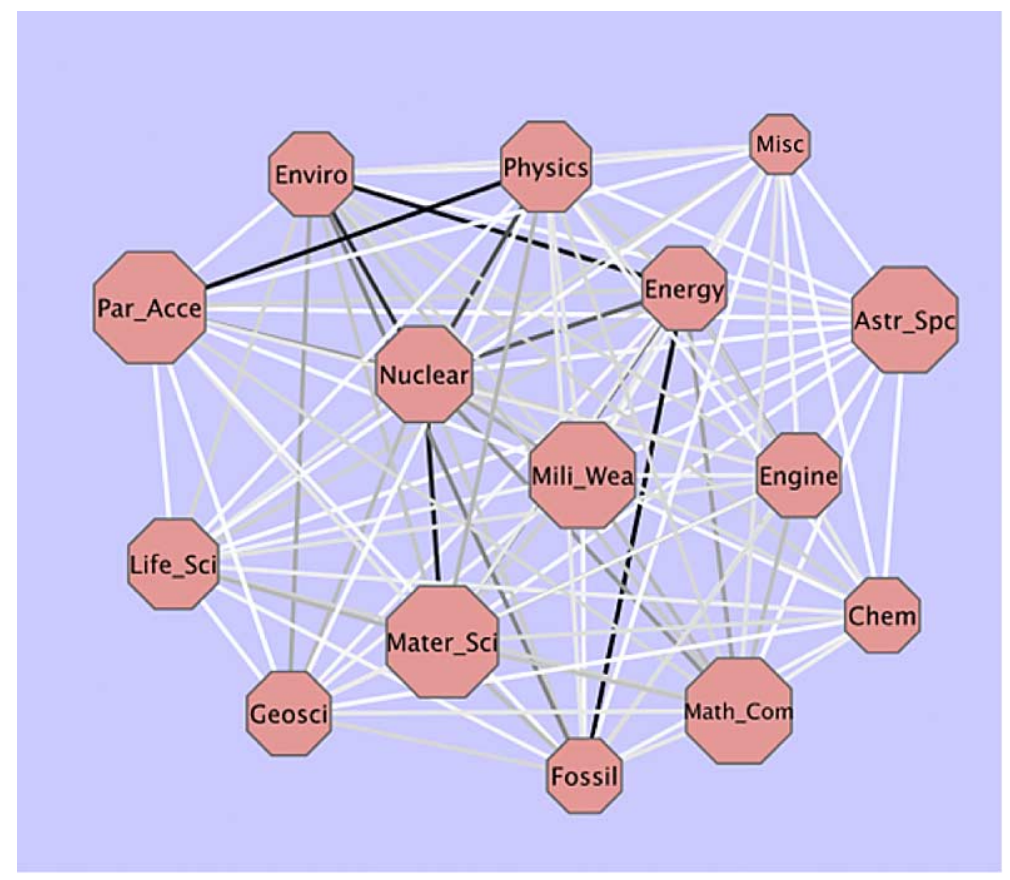

Fig. 6. Social network of subject fields during the time period 2000-2012. (Colors are visible in the online version of the article; http://dx.doi.org/10.3233/ISU-130715.)

This visualization of social networks makes node interaction easy to interpret. However, there are other important statistics to interpret for network structures. In this study we use two of the network metrics: betweenness centrality and closeness centrality for weighted networks, where the weights are the frequencies in Table 2. These statistics are calculated using $\mathrm{R}$ software and the thet package of Opsahl [17]. The results are shown in Table 3.

Looking at Table 3, the non-zero betweenness centrality values for the time period of 1980-2000 indicates that only three of the subject fields - nuclear (0.28), environment $(0.20)$ and energy (0.13) play a key role by being in the middle of the network. These three subject fields bring the scientists together in their scientific collaboration. Further, by being in the middle of the network, they continue to retain their importance during the time period of 2000-2012. During this period, physics and engineering subject fields gain importance in terms of collaboration by judging on their small betweenness centrality as compared to zero betweenness centrality during the years of 1980-2000. This shows that physics and engineering subject field also play a more important role in collaboration during the last 12 years.

Table 3 further shows that astronomy and space, along with chemistry, are the two subject fields with the smallest closeness centrality statistics for both time periods under consideration. This indicates that these subject fields are more isolated in the network with respect to levels of collaboration. The subject fields of nuclear, energy, environment, and material science have the largest closeness centrality values. This indicates that these subject fields are more open to collaboration in comparison to other subject fields with smaller closeness centrality. Another prominent feature in Table 3 is that the closeness centrality value of material science jumps from the fifth to the third highest closeness centrality in the last 12 years as compared to the time period 1980-2000. This indicates that the role of material scientists in energy projects has grown in the last 12 years. 
Table 3

Betweenness and closeness centrality values (1920-2012)

\begin{tabular}{lcccc}
\hline Subject fields & \multicolumn{2}{c}{ Betweenness centrality } & \multicolumn{2}{c}{ Closeness centrality } \\
\cline { 2 - 4 } & $1980-2000$ & $2000-2012$ & 0.038 & $2000-2012$ \\
\hline Astronomy and space & 0.00 & 0.00 & 0.045 & 0.034 \\
Chemistry & 0.00 & 0.00 & 0.069 & 0.047 \\
Energy & 0.13 & 0.17 & 0.057 & 0.106 \\
Engineering & 0.00 & 0.02 & 0.073 & 0.076 \\
Environment & 0.20 & 0.00 & 0.065 & 0.099 \\
Fossil fuels & 0.00 & 0.00 & 0.044 & 0.086 \\
Geoscience & 0.00 & 0.00 & 0.049 & 0.061 \\
Life sciences & 0.00 & 0.00 & 0.061 & 0.055 \\
Material science & 0.00 & 0.08 & 0.055 & 0.099 \\
Math and computation & 0.00 & 0.00 & 0.052 & 0.077 \\
Military and weapon & 0.00 & 0.00 & 0.010 & 0.063 \\
Miscellaneous & 0.00 & 0.00 & 0.074 & 0.041 \\
Nuclear & 0.28 & 0.24 & 0.047 & 0.110 \\
Particle acceleration & 0.00 & 0.00 & 0.061 & 0.070 \\
Physics & 0.00 & 0.06 & 0.084 \\
\hline
\end{tabular}

\section{Conclusions}

In this study we analyzed the levels of scientific collaboration between different subject fields using the OSTI database and the social network modeling tools. Results show an increasing collaboration between scientists for almost all subject fields included in the OSTI database. We determined that the productivity among scientists has increased in the last 12 years.

By bringing all the subject fields together in a social network, we analyzed the interaction between the subject fields. We used authors having publications in more than one subject field as bridges connecting subject fields to each other. In doing so, we constructed two separate social networks for the time periods of 1980-2000 and 2000-2012 to determine structural changes in the network model between subject fields. Our results show that the nuclear, energy, and environment subject fields play a key role in the network with respect to level of scientific collaboration. These results support the increasing trend in science to look for renewable energy sources as alternative of fossil fuel based energy sources. At this point, nuclear energy research is also still very attractive topic although requires environmental research accompanying with it.

Another interesting finding in this study is that there is an increasing scientific collaboration between the subject fields, such as particle acceleration-physics, nuclear-energy and nuclear-physics. On the other hand, the level of collaboration between environment-fossil fuels has decreased in the last 12 years as compared to results during 1980-2000 based on the technical reports done for DOE. Note that, this does not mean that there is a decreased collaboration between these fields overall. This might be due to any changes in energy policies and interest of DOE.

In summary, when evaluating the overall results, we see that there are three subject fields - nuclear, energy and environment - that are at the center of scientific collaboration, bringing scientists together in common scientific projects. This is not surprising, since DOE's focus has recently been in these areas and there is an increasing interest in renewable energy research. 
In conclusion, it would be interesting to study whether there is a correlation between the level of scientific collaboration and the amount of funds awarded in these subject fields, as well as the impact of funding on the level productivity among scientists. This can serve as a platform for future funding in different subject fields.

\section{Acknowledgements}

The first author acknowledges the support of EPSCoR Grant Award IIA-IIa-5112-470 from DOE for three years as a Lead Researcher on the grant. Second author expresses his gratitude to Prof. Bozdogan as a Post-Doc and Research Associate on the grant. We also extend our thanks to Mr. William Thompson IV, Graduate Research Assistant on the grant, for his assistance in cleaning and providing the original OSTI data set for analysis. We express our gratitude to Mr. Ed Coppock who managed the EPSCoR Grant Award wonderfully. Finally, comments of two anonymous reviewers are acknowledged and appreciated which improved the presentation of this paper.

\section{References}

[1] S. Bandyopadhyay, A.R. Rao and B.K. Sinha, Models for Social Networks with Statistical Applications, SAGE Publications, 2010.

[2] A. Barrat, M. Barthelemy, R. Pastor-Satorras and A. Vespignani, The architecture of complex weighted networks, Proceedings of the National Academy of Sciences 101(11) (2004), 3747-3752.

[3] A. Bavelas, Communication patterns in task oriented groups, Journal of Acoustical Society of America 22 (1950), 271-282.

[4] P. Bonacich, Power centrality: a family of measures, American Journal of Sociology 92 (1987), 1170-1182.

[5] S.P. Borgatti, Centrality and network flow, Social Networks 27(1) (2005), 55-71.

[6] U. Brandes, A faster algorithm for betweenness centrality, The Journal of Mathematical Sociology 25 (2001), $163-177$.

[7] T. Cserpes, Measuring identity change: Analysing fragments from the diary of Sandor Karolyi with social-network analysis, European Review of History 19(5) (2012), 729-748.

[8] Department of Energy (DOE) Office of Scientific and Technical Information, Information Bridge: DOE Scientific and Technical Information, 2012, available at: http://www.osti.gov/bridge/ (accessed January 4, 2013).

[9] M. Eblen, R.R. Fabsitz, J.L. Olson, K. Pearson, L.R. Pool, M. Puggal, C. Wu and R.M. Wagner, Social network analysis comparing researcher collaborations in two cardiovascular cohort studies, Research Evaluation 21 (2012), 392-405.

[10] L.C. Freeman, Centrality in social network conceptual clarification, centrality in social networks conceptual clarification, Social Networks 1 (1978/1979), 215-239.

[11] L. Hossain and D. Fazio, The social networks of collaborative process, Journal of High Technology Management Research 20 (2009), 119-130.

[12] R. Johnson, B. Kovacs and A. Vicsek, A comparison of email networks and off-line social networks: A study of a mediumsized bank, Social Networks 34 (2012), 462-469.

[13] E.D. Mainas, The analysis of criminal and terrorist organisations as social network structures: A quasi-experimental study, International Journal of Police Science \& Management 14(3) (2012), 264-282.

[14] S. Maslow and K. Sneppen, Specificity and stability in topology of protein networks, Science 296 (2002), $910-913$.

[15] M.E.J. Newman, Scientific collaboration networks. II. Shortest paths, weighted networks, and centrality, Physical Review E64 (2001), 016132.

[16] M.E.J. Newman, Analysis of weighted networks, Physical Review E70 (2004), 056131.

[17] T. Opsahl, Structure and evolution of weighted networks, University of London, 2009, available at: http://toreopsahl.com/ publications/thesis/ (accessed January 4, 2013).

[18] T. Opsahl, F. Agneessens and J. Skvoretz, Node centrality in weighted networks: Generalizing degree and shortest paths, Social Networks 32 (2010), 245-251.

[19] J. Oshima, R. Oshima and Y. Matsuzawa, Knowledge building discourse explorer: a social network analysis application for knowledge building discourse, Educational Technology Research and Development 60(5) (2012), 903-921.

[20] J. Pow, K. Gayen, L. Elliott and R. Raeside, Understanding complex interactions using social network analysis, Journal of Clinical Nursing 21 (2012), 2772-2779. 
[21] A. Quinn, R. Woehler and K. Tiemann, Social network analysis for analyzing groups as complex systems, Journal of Social Service Research 38 (2012), 605-618.

[22] K. Schneider, C. Rainwater, E. Pohl, I. Hernandez and J.E. Ramirez-Marquez, Social network analysis via multi-state reliability and conditional influence models, Reliability Engineering and System Safety 109 (2013), 99-109.

[23] P. Shannon, A. Markiel, O. Ozier, N.S. Baliga, J.T. Wang, D. Ramage, N. Amin, B. Schwikowski and T. Ideker, Cytoscape: A software environment for integrated models of biomolecular interaction networks, Genome Research 13 (2003), 2498-2504.

[24] N.M. Tichy, M.L. Tushman and C. Fombrun, Social network analysis for organizations, The Academy of Management Review 4(4) (1979), 507-519.

[25] S. Wasserman and K. Faust, Social Network Analysis: Methods and Applications, Cambridge Univ. Press, 1994.

[26] S. Wassermann and D. Steinley, Sensitivity analysis of social network data and methods: some preliminary results, in: Dynamic Social Network Modeling and Analysis, National Academies Press, Washington, 2003.

[27] Wikipedia contributors, Social Structure. Wikipedia, The Free Encyclopedia, available at: http://en.wikipedia.org/wiki/ Social_structure (accessed January 4, 2013).

[28] Wikipedia contributors, Dyad, Wikipedia, The Free Encyclopedia, available at: http://en.wikipedia.org/wiki/ Dyad_(sociology) (accessed January 4, 2013). 